\title{
PAPER
}

\section{Cerebrovascular pressure reactivity is related to global cerebral oxygen metabolism after head injury}

\author{
L A Steiner, J P Coles, M Czosnyka, P S Minhas, T D Fryer, F I Aigbirhio, J C Clark, \\ P Smielewski, D A Chatfield, T Donovan, J D Pickard, D K Menon
}

J Neurol Neurosurg Psychiatry 2003;74:765-770

See end of article for authors' affiliations

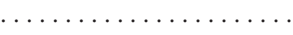

Correspondence to: Dr Luzius A Steiner, Academic Neurosurgery, Box 167, Addenbrooke's Hospital, Cambridge CB2 2QQ, UK; las30@cam.ac.uk

Received 7 August 2002 In revised form

29 January 2003

Accepted

4 February 2003

\begin{abstract}
Background: After head injury, impaired cerebrovascular autoregulation has been associated with abnormally high or low cerebral blood flow. The physiological relevance of cerebral blood flow levels is difficult to assess in these patients, whose cerebral metabolic rate for oxygen $\left(\mathrm{CMRO}_{2}\right)$ is known to be abnormal. Investigation of these relations requires quantitative measures of cerebral blood flow and $\mathrm{CMRO}_{2}$, to allow assessment of oxygen supply and demand relations.

Objectives: To investigate the relation between dysautoregulation and global cerebral oxygen metabolism following head injury.

Methods: Using positron emission tomography, global cerebral blood flow, $\mathrm{CMRO}_{2}$, and oxygen extraction fraction were determined in 22 patients who were investigated in 26 examinations on days 1 to 11 (mean (SD), 3.5 (2.3)) after head injury. Cerebrovascular pressure reactivity was assessed using a pressure reactivity index, calculated as the moving linear correlation coefficient between mean arterial blood pressure and intracranial pressure. Outcome was assessed six months after injury using the Glasgow outcome scale.

Results: Low $\mathrm{CMRO}_{2}$ was associated with disturbed pressure reactivity (inverse function, $R^{2}=0.21$, $p=0.018$ ) and there was a correlation between disturbed pressure reactivity and oxygen extraction fraction (quadratic function, $R^{2}=0.55, \mathrm{p}=0.0001$ ). There was no significant relation between pressure reactivity and cerebral blood flow. An unfavourable outcome was associated with disturbed pressure reactivity. There was no significant relation between outcome and $\mathrm{CMRO}_{2}$ or oxygen extraction fraction.

Conclusions: There is a close relation between dysautoregulation and abnormal cerebral metabolism but not blood flow. Further studies are needed to determine whether metabolic dysfunction is a result of or a cause of disturbed pressure reactivity, and to establish if there is a relation between cerebral oxygen metabolism and outcome.
\end{abstract}

$\mathrm{T}$ he mechanisms that lead to post-traumatic cerebrovascular dysautoregulation are incompletely understood. In animals, vasoparalysis caused by ischaemia has been shown to abolish autoregulation. ${ }^{1}$ Several other mechanisms leading to ischaemia-such as compression of blood vessels by astrocyte swelling, ${ }^{2}$ vasospasm, ${ }^{3}$ or erythrocyte and leucocyte stasis ${ }^{4}$-have been suggested as causes of dysautoregulation. While other possible mechanisms involve free radicals, ${ }^{5}$ newer work has focused on the role of potassium channel dysfunction, ${ }^{67}$ endothelin, and nitric oxide ${ }^{8}$ in autoregulatory failure.

Although the evidence is not unequivocal, several studies have documented an association between disturbed autoregulation and unfavourable outcome after head injury. ${ }^{9-12}$ Despite the fact that dysautoregulation is a result of a multitude of disturbances, it seems reasonable to explain the association of intact autoregulation with good outcome by protection of the injured brain against ischaemia and hyperaemia. There is indirect support for this hypothesis, as Overgaard and Tweed ${ }^{9}$ have shown that in head injured patients low and high cerebral blood flow are associated with impaired autoregulation. While low and high cerebral blood flow have been equated with ischaemia and hyperaemia, such inferences are uncertain in head injured patients, who may show primary changes in metabolism ${ }^{13}$ or be treated with drugs such as propofol and barbiturates or hypothermia. In this setting, measurements of the cerebral metabolic rate for oxygen $\left(\mathrm{CMRO}_{2}\right)$ and oxygen extraction fraction are necessary to make the distinction between ischaemia and hyperaemia on the one hand, and metabolically coupled hypoperfusion or hyperperfusion on the other. Decisions regarding treatment in patients with impaired autoregulation would be facilitated by an understanding of the physiological context in which dysautoregulation is observed. For example, if dysautoregulation were exclusively observed in patients with ischaemia caused by a low cerebral perfusion pressure, blood pressure augmentation could restore normal physiology. On the other hand, if the prominent association of dysautoregulation were with hyperaemia and vasoparalysis, therapeutic strategies would need to be significantly different.

Investigation of these relations requires methods that provide quantitative measures of cerebral blood flow and oxygen metabolism, to allow assessment of the balance of oxygen supply and demand, and enable robust inferences to be made about the presence of ischaemia and hyperaemia. We have used positron emission tomography (PET) to measure cerebral blood flow, $\mathrm{CMRO}_{2}$, and oxygen extraction fraction, and have compared these variables with an index of cerebrovascular pressure reactivity as a measure of autoregulatory efficiency. The method we used for determination of pressure reactivity calculates a pressure reactivity index (PRx) based on the analysis of spontaneous slow waves of mean arterial blood pressure and intracranial pressure. ${ }^{14}$ Our aim in this study was to test the hypothesis that after head injury, disturbed

Abbreviations: $\mathrm{CMRO}_{2}$, cerebral metabolic rate for oxygen; $\mathrm{GCS}$, Glasgow coma scale; GOS, Glasgow outcome scale; PRx, pressure reactivity index 


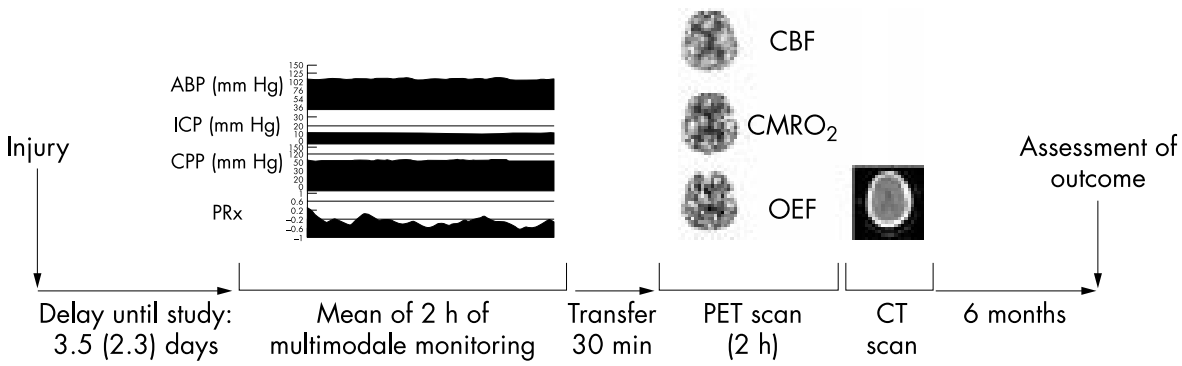

Figure 1 Schematic illustration of the study protocol. $\mathrm{ABP}$, arterial blood pressure; $\mathrm{CBF}$, cerebral blood flow; $\mathrm{CMRO}_{2}$, cerebral metabolic rate for oxygen; CPP, cerebral perfusion pressure; CT Scan, computed tomography scan for coregistration; ICP, intracranial pressure; OEF, oxygen extraction fraction; PET, positron emission tomography; PRx, pressure reactivity index.

autoregulation is related to disturbed oxygen metabolismthat is, low $\mathrm{CMRO}_{2}$ and oxygen extraction fraction outside the physiological range rather than low or high absolute values of cerebral blood flow.

\section{METHODS}

The local research ethics committee approved the study, and informed assent was obtained from the next of kin of all investigated patients.

\section{Patients and clinical management}

Between January 1998 and June 2001, we undertook 36 PET examinations in 29 head injured patients who had been admitted to our neurosciences critical care unit and who had continuous monitoring of cerebral pressure reactivity. No patient had more than two PET scans.

All patients were managed with protocol driven treatment aimed at maintaining intracranial pressure below $20 \mathrm{~mm} \mathrm{Hg}$ and cerebral perfusion pressure above $70 \mathrm{~mm} \mathrm{Hg}$. Graded interventions included sedation (propofol, fentanyl) and neuromuscular blockade, rapid surgery for space occupying lesions, drainage of CSF, dopamine or noradrenaline (norepinephrine) for control of cerebral perfusion pressure, mannitol, mild hyperventilation (to a $\mathrm{P}_{\mathrm{a}} \mathrm{CO}_{2}$ of $\sim 4.5 \mathrm{kPa}$ ), mild to moderate hypothermia $\left(33-36^{\circ} \mathrm{C}\right)$, decompressive craniectomy, and barbiturate coma. A single patient in whom thiopentone (thiopental) had been given during PET was excluded from further analysis. No patient received mannitol in the six hours before PET. Outcome was assessed six months after injury using the Glasgow outcome scale (GOS), ${ }^{15}$ with dichotomisation of patients into favourable (good outcome and moderately disabled) and unfavourable (dead, persistent vegetative state, and severely disabled) outcome groups.

\section{Quantification of autoregulatory efficiency using a pressure reactivity index}

We used measurements of PRx to assess cerebrovascular pressure reactivity in this group of patients. This method has been described in detail elsewhere. ${ }^{14}$ Briefly, for calculation of PRx, the analogue signals of invasive arterial blood pressure monitors and intraparenchymal intracranial pressure transducers were sampled and digitised. Mean arterial blood pressure and intracranial pressure values were calculated over six second intervals using waveform time integration. PRx was calculated every 60 seconds as the moving linear correlation coefficient between the last 40 consecutive six second averages of arterial blood pressure and intracranial pressure. Possible values for PRx therefore range from -1 to +1 . Negative or zero values indicate intact pressure reactivity, while increasing positive values indicate increasingly disturbed pressure reactivity. PRx values greater than 0.2 indicate severely disturbed autoregulation and are associated with a poorer outcome. ${ }^{14}$ Data analysed in this paper were based on an averaged measurement of PRx over the two hours immediately preceding the PET scan.
Following the two hour period of PRx recording, patients were transported to the PET scanning suite and stabilised before imaging studies were conducted. During acquisition of PRx and PET imaging, haemodynamic stability was ensured by titrating administration of intravenous fluids and if necessary vasoactive agents. To avoid comparing data acquired from different points on a patient's autoregulatory curve, cerebral perfusion pressure during PET scanning was kept as close as possible to the perfusion pressure measured during PRx acquisition. Nine examinations in which the difference in mean perfusion pressure during PET scanning and PRx acquisition exceeded $10 \mathrm{~mm} \mathrm{Hg}$ were excluded from analysis. The rates of the sedative infusion were left unchanged during acquisition of PRx data and PET imaging. The ventilator settings were adjusted to keep arterial partial pressure of $\mathrm{CO}_{2}$ $\left(\mathrm{PaCO}_{2}\right)$ constant. The study protocol is illustrated in fig $\mathrm{l}$.

\section{Positron emission tomography}

PET studies were undertaken on a General Electric Advance scanner (GE Medical Systems, Milwaukee, Wisconsin, USA). A 10 minute transmission scan using two rotating ${ }^{68} \mathrm{Ge} /{ }^{68} \mathrm{Ga}$ rods was done in all patients and used to correct subsequent emission scans for photon attenuation. Emission data were acquired during a steady state infusion of $800 \mathrm{MBq}$ of $\mathrm{H}_{2}{ }^{15} \mathrm{O}$, following a 60 second inhalation of $300 \mathrm{MBq}$ of $\mathrm{C}^{15} \mathrm{O}$, and during a steady state inhalation of $7200 \mathrm{MBq}$ of ${ }^{15} \mathrm{O}_{2}$. Images were reconstructed using the PROMIS 3D filtered back projection algorithm. ${ }^{16}$ The emission data were coregistered to spiral computed tomography (CT) images obtained immediately after each PET scan. Parametric maps of cerebral blood flow, cerebral blood volume, $\mathrm{CMRO}_{2}$, and oxygen extraction fraction were calculated by inputting simultaneous PET and arterial tracer activity measurements into standard models, using a capillary to large vessel packed cell volume ratio of 0.85 , and a partition coefficient for $\mathrm{H}_{2}{ }^{15} \mathrm{O}$ of $0.95 .{ }^{17}{ }^{18}$ Images were analysed using custom designed software, incorporating elements of several software packages, including Statistical Parametric Mapping (SPM99, Wellcome Department of Cognitive Neurology, London, UK; http://www.fil.ion.bpmf.ac.uk/spm/), Matlab 5.2 (MathWorks Inc, Natick, Maryland, USA), Analyze 4.0 (AnalyzeDirect Inc, Lenexa, Kansas, USA), and registration by multiresolution optimisation of mutual information (mpr, Department of Radiological Sciences, Guys Hospital, London, $\left.\mathrm{UK}^{19}{ }^{20}\right)$. Individual CT images were edited manually to extract a template that identified brain tissue excluding extracranial tissue, cerebrospinal fluid, and extra-axial haematomas. This brain template was applied to the spatially coregistered PET images, thus restricting analysis to brain tissue. From these data, the global mean values for cerebral blood flow, $\mathrm{CMRO}_{2}$, and oxygen extraction fraction were calculated.

\section{Statistical analysis}

Statistical analysis was done using SPSS 10.1 for Windows (SPSS Inc, Chicago, Illinois, USA). Regression methods were used to investigate correlations between PRx and global mean 
Table 1 Individual patient data

\begin{tabular}{|c|c|c|c|c|c|c|c|c|c|c|c|}
\hline Patient & Day & GCS & $\begin{array}{l}\text { Marshall } \\
\text { score }\end{array}$ & PRx & $\begin{array}{l}\text { CPP PRx (mm } \\
\mathrm{Hg})\end{array}$ & $\begin{array}{l}\text { CPP PET } \\
(\mathrm{mm} \mathrm{Hg})\end{array}$ & $\begin{array}{l}\mathrm{ICP}(\mathrm{mm} \\
\mathrm{Hg})\end{array}$ & OEF (\%) & $\begin{array}{l}\mathrm{CMRO}_{2} \\
\left(\mathrm{ml} .100 \mathrm{ml}^{-1} \cdot \mathrm{min}^{-1}\right)\end{array}$ & $\begin{array}{l}\text { CBF } \\
\left(\mathrm{ml} .100 \mathrm{ml}^{-1} \cdot \mathrm{min}^{-1}\right)\end{array}$ & GOS \\
\hline la & 1 & 12 & NEML & -0.172 & 81 & 75 & 23 & 59.2 & 105.9 & 26.5 & 4 \\
\hline $1 b$ & 3 & & & -0.094 & 77 & 73 & 29 & 35.3 & 66.3 & 27.9 & \\
\hline 2 & 3 & 8 & NEML & -0.085 & 76 & 76 & 22 & 51.2 & 79.1 & 28.3 & 4 \\
\hline 3 & 2 & 8 & DII & -0.090 & 77 & 71 & 15 & 40.5 & 70.4 & 30.6 & 5 \\
\hline 4 & 7 & 6 & DII & 0.409 & 89 & 83 & 25 & 27.8 & 49.0 & 23.4 & 1 \\
\hline $5 a$ & 1 & 5 & EML & 0.136 & 80 & 74 & 3 & 45.0 & 63.5 & 23.0 & 4 \\
\hline $5 b$ & 5 & & & -0.031 & 77 & 68 & 10 & 46.7 & 88.1 & 31.4 & \\
\hline $6 a$ & 2 & 9 & EML & -0.127 & 76 & 75 & 30 & 37.9 & 84.7 & 36.7 & 5 \\
\hline $6 b$ & 5 & & & -0.068 & 71 & 75 & 20 & 37.1 & 77.0 & 35.6 & \\
\hline 7 & 2 & 4 & NEML & 0.634 & 78 & 79 & 17 & 27.3 & 71.7 & 41.2 & 1 \\
\hline 8 & 5 & 4 & NEML & -0.208 & 79 & 72 & 11 & 42.0 & 78.6 & 29.3 & 3 \\
\hline 9 & 5 & 12 & DII & -0.032 & 89 & 98 & 14 & 34.9 & 73.3 & 28.1 & 5 \\
\hline 10 & 2 & 8 & EML & -0.122 & 68 & 70 & 14 & 38.3 & 100.1 & 54.4 & 5 \\
\hline 11 & 3 & 7 & NEML & -0.151 & 80 & 86 & 15 & 37.7 & 74.4 & 32.1 & 4 \\
\hline 12 & 4 & 9 & NEML & -0.198 & 87 & 83 & 25 & 45.3 & 69.7 & 21.3 & 5 \\
\hline 13 & 2 & 7 & NEML & -0.142 & 78 & 73 & 11 & 52.3 & 91.3 & 28.2 & 4 \\
\hline 14 & 2 & 13 & EML & 0.089 & 74 & 69 & 14 & 41.5 & 83.8 & 34.9 & 5 \\
\hline 15 & 11 & 3 & NEML & 0.134 & 85 & 90 & 9 & 32.1 & 56.8 & 31.0 & 3 \\
\hline 16 & 3 & 8 & DII & -0.127 & 72 & 75 & 14 & 42.1 & 88.9 & 28.3 & 5 \\
\hline 17 & 7 & 6 & NEML & -0.063 & 95 & 98 & 22 & 40.9 & 81.7 & 29.2 & 3 \\
\hline 18 & 5 & 6 & EML & -0.032 & 78 & 82 & 33 & 32.3 & 78.8 & 42.4 & 3 \\
\hline 19 & 3 & 12 & NEML & -0.176 & 84 & 86 & 21 & 46.7 & 76.6 & 28.1 & 3 \\
\hline 20 & 3 & 8 & DII & 0.151 & 87 & 89 & 14 & 31.6 & 68.9 & 30.7 & 3 \\
\hline $21 a$ & 1 & 10 & NEML & 0.007 & 69 & 72 & 20 & 45.9 & 87.9 & 34.0 & 3 \\
\hline $21 \mathrm{~b}$ & 3 & & & 0.003 & 78 & 73 & 22 & 60.2 & 103.0 & 26.8 & \\
\hline 22 & 3 & 12 & EML & 0.044 & 67 & 68 & 18 & 29.9 & 67.1 & 43.6 & 5 \\
\hline
\end{tabular}

DIl, diffuse injury II; Day, day after injury; CBF, global cerebral blood flow; $\mathrm{CMRO}_{2}$, global cerebral metabolic rate for oxygen; CPP PET, mean cerebral perfusion pressure during PET scan; CPP PRx, mean cerebral perfusion pressure during PRx acquisition; EML, evacuated mass lesion; GCS, Glasgow coma scale score at admission; GOS, Glasgow outcome scale (1 = dead, $3=$ severely disabled, 4 = moderately disabled, $5=$ good recovery); ICP, intracranial pressure; NEML, non-evacuated mass lesion; OEF, global oxygen extraction fraction; PRx, pressure reactivity index.

values of cerebral blood flow, $\mathrm{CMRO}_{2}$, and oxygen extraction fraction. Multiple sampling (four patients were studied twice) was not corrected for, as the interval between these studies was more than 48 hours and with such a delay it is reasonable to assume that the states of autoregulation and cerebral blood flow and oxygen metabolism were independent. For comparisons between outcome and other variables, one way analysis of variance and Spearman rank correlations were used. Numerical data are presented as mean (SD).

\section{RESULTS}

Data from 26 examinations in 22 patients were available for analysis. Patients were aged 17 to 64 years (mean 33 (13) years), and their postresuscitation Glasgow coma scale (GCS) score ranged from 3 to 13 (median 8). PET scans were performed one to 11 (mean $3.5(2.3)$ ) days after injury. There were only two patients with marked disturbance of pressure reactivity $(\mathrm{PRx}>0.2)$. Mean values for global cerebral blood flow, $\mathrm{CMRO}_{2}$, and oxygen extraction fraction were 31.8 (7.2) $\mathrm{ml} .100 \mathrm{ml}^{-1} \cdot \mathrm{min}^{-1} \quad$ (range 21.3 to 54.4 ), 78.3 (13.5) $\mu \mathrm{mol} .100 \mathrm{ml}^{-1} \cdot \mathrm{min}^{-1}$ (49.0 to 105.9 ), and 40.8 (8.7)\% (27.3\% to $60.2 \%)$, respectively. Individual patient data including Marshall score ${ }^{21}$ are presented in table 1 . There were no significant correlations between cerebral perfusion pressure and any of the PET derived variables. Cerebral blood flow was correlated with oxygen extraction fraction $(R=-0.40, \mathrm{p}=0.04)$ but not with $\mathrm{CMRO}_{2}$. Oxygen extraction fraction was also correlated with $\mathrm{CMRO}_{2}(R=0.71, \mathrm{p}<0.0001)$. There were no significant correlations between GCS scale and any of the variables measured by PET or between GCS and Prx.

While some patients with positive PRx values showed low global cerebral blood flow, there was substantial interindividual variation (table 1 ), and there was no significant correlation between cerebral blood flow and PRx (fig 2). The relation between $\mathrm{CMRO}_{2}$ and $\mathrm{PRx}$ was characterised by an inverse function $\left(R^{2}=0.21, \mathrm{p}=0.018\right)$, showing that a very low $\mathrm{CMRO}_{2}$ in these sedated head injured patients is associated with disturbed pressure reactivity, and that higher $\mathrm{CMRO}_{2}$ is associated with better pressure reactivity (fig 3). However, the $\mathrm{CMRO}_{2}$ relation does not allow us to determine whether the low $\mathrm{CMRO}_{2}$ values associated with disturbed pressure reactivity were the result of ischaemia or primary metabolic failure. This question was addressed by studying the relation between oxygen extraction fraction and PRx, which can be characterised by a quadratic function $\left(R^{2}=0.55, \mathrm{p}=0.0001\right)$. This model suggests that both low oxygen extraction fraction (representing luxury perfusion, hyperaemia, or necrotic tissue) and high oxygen extraction fraction (representing misery perfusion or ischaemia) are associated with disturbed pressure reactivity (fig 4 ).

\section{Outcome}

Outcome data were available for all 22 patients. Those with unfavourable outcome had worse pressure reactivity (PRx $0.09(0.28) v-0.09$ (0.08); $\mathrm{p}=0.035)$ and a higher mean cerebral perfusion pressure (84 (7) $v 77$ (7) $\mathrm{mm} \mathrm{Hg}$; $=0.030)$. There was a trend for oxygen extraction fraction and $\mathrm{CMRO}_{2}$ to

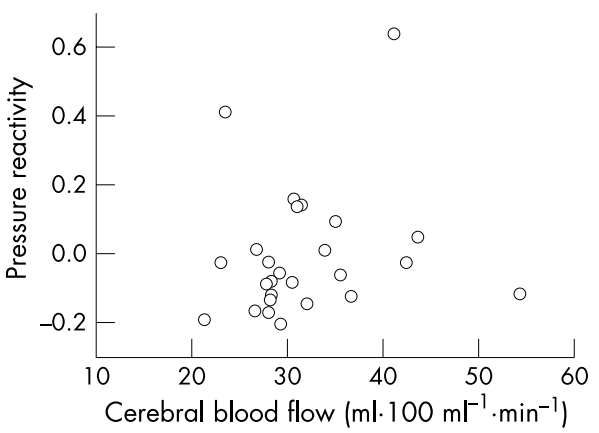

Figure 2 Relation between global cerebral blood flow and pressure reactivity (pressure reactivity index). There is no significant relation between global cerebral blood flow and pressure reactivity index in this group of patients, with cerebral blood flow predominantly in the low to normal range. 


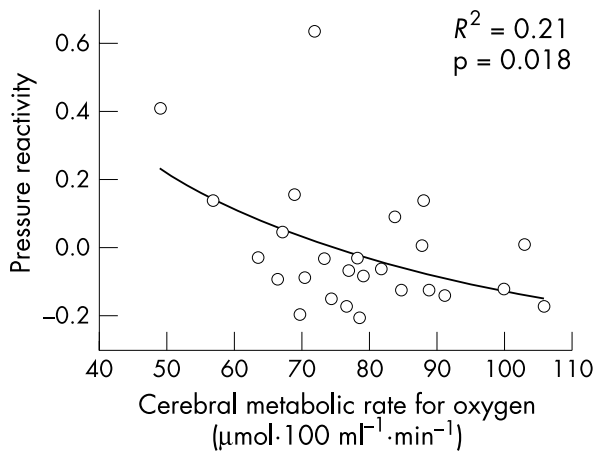

Figure 3 Relation between global cerebral metabolic rate for oxygen and pressure reactivity (pressure reactivity index). The distribution is characterised by an inverse function. Very low global cerebral metabolic rate for oxygen $\left(\mathrm{CMRO}_{2}\right)$ in these sedated head injured patients is associated with disturbed pressure reactivity and higher $\mathrm{CMRO}_{2}$ is associated with better pressure reactivity.

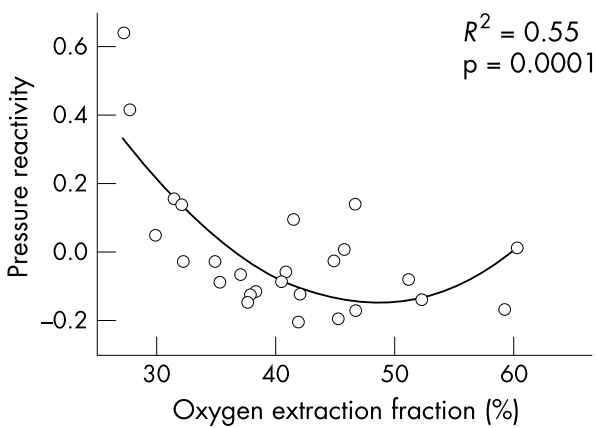

Figure 4 Relation between global oxygen extraction fraction and pressure reactivity (pressure reactivity index). A quadratic function represents the best fit to the data. The model suggests that both low oxygen extraction fraction (representing luxury perfusion, hyperaemia, or necrotic tissue) and high oxygen extraction fraction (representing misery perfusion or ischaemia) are associated with disturbed pressure reactivity.

be lower in patients with an unfavourable outcome $(38.7 \% \mathrm{v}$ $42.2 \%$ and $75.2 \vee 80.2 \mu \mathrm{mol} .100 \mathrm{ml}^{-1} \cdot \mathrm{min}^{-1}$, respectively) but the differences did not reach statistical significance. The lowest values for oxygen extraction fraction were found in the two patients who died. There were no significant Spearman rank correlations between GOS and PRx $(R=-0.34 ; \mathrm{p}=0.13)$, cerebral blood flow $(R=0.25 ; \mathrm{p}=0.27), \mathrm{CMRo}_{2}(R=0.31$; $\mathrm{p}=0.16)$, or oxygen extraction fraction $(R=0.17 ; \mathrm{p}=0.46)$.

\section{DISCUSSION}

Our results show a close relation between pressure reactivity, global $\mathrm{CMRO}_{2}$, and oxygen extraction fraction. However, in contrast to earlier studies, ${ }^{9}$ we were unable to demonstrate a relation between measures of autoregulatory efficiency and cerebral blood flow.

\section{Methodological issues}

$\mathrm{PRx}$ and $\mathrm{CMRO}_{2}$ were clearly related statistically. However, several questions need to be addressed to determine the mechanistic and clinical significance of this relation. It is important to exclude the possibility that the association between $\mathrm{CMRO}_{2}$ and PRx reflected the fact that both low $\mathrm{CMRO}_{2}$ and high PRx are associated with a low GCS-CMRo, has been shown to correlate with the GCS, ${ }^{22}$ and earlier work has also shown a correlation between PRx and the GCS. ${ }^{14}$ There are several important differences between these earlier studies and the present data which argue against our relation simply being a reflection of GCS. First, we found no relation between GCS and $\mathrm{CMRO}_{2}$ in our data and, in contrast to earlier studies, ${ }^{22}$ all our patients were sedated with propofol during measurement of $\mathrm{CMRO}_{2}$. Further, in the data presented here as well as in a recent series of more than 100 patients ${ }^{23}$ we found no correlation between GCS and PRx. Interestingly, in the same series of patients there was no correlation between GCS and GOS, possibly reflecting improved prehospital care.

Could our results have been a result of sedation or other confounding variables such as $\mathrm{PaCO}_{2}$ or changes in vasoactive drug infusion rates? The wide range of $\mathrm{CMRO}_{2}$ values that we observed despite the fact that all the patients received similar doses of propofol (3-5 mg. $\mathrm{kg}^{-1} \cdot \mathrm{h}^{-1}$ ), suggests that sedation with propofol was unlikely to have been the cause of $\mathrm{CMRO}_{2}$ variation between patients. It also seems unlikely that sedation could have been responsible for dysautoregulation, as cerebrovascular autoregulation is well preserved even at much higher doses of propofol. ${ }^{24}$ Other variables such as $\mathrm{PaCO}_{2}$ or temperature were carefully controlled. Infusion rates of dopamine and noradrenaline were adjusted to ensure stability of cerebral perfusion pressure in some patients. There are no data on the potential effects of this on cerebrovascular resistance and therefore autoregulation or, in the case of blood brain-barrier breakdown, on cerebral metabolism through a $\beta$ adrenergic effect. However, the necessary changes in dose were very small and we chose to adjust the drug rates rather than to tolerate changes in cerebral perfusion pressure that would have made the comparison between PET and autoregulation impossible.

While the association of $\mathrm{CMRO}_{2}$ with $\mathrm{PRx}$ is intriguing, these data do not provide a complete explanation of the underlying physiology, as low $\mathrm{CMRo}_{2}$ values may be a result of critical ischaemia or primary metabolic failure. The quadratic function fitted to our oxygen extraction fraction $v$ PRx plot (fig 4) suggests that autoregulation may be impaired in the setting of both ischaemia and hyperaemia, implying that ischaemia and metabolic dysfunction may both provide the substrate for dysautoregulation. Whereas there is no doubt that hyperaemia is associated with loss of autoregulation, there were no patients with relevant global ischaemia represented by a high oxygen extraction fraction in this series, and only eight of our patients had a PRx value above zero. Of those, only two had severely disturbed autoregulation ( $\mathrm{PRx}>0.2)$. Therefore, our results-that is, the fitted quadratic function-may be inappropriately influenced by the small number of patients and the location of individual data points.

It is possible to characterise the relation between PRx and oxygen extraction fraction with a linear or inverse function. Based on the fact that it has been shown in animals that ischaemia does lead to loss of autoregulation, ${ }^{1}$ it is reasonable to expect a high global oxygen extraction fraction to be associated with a high PRx. However, our data do not allow a definitive conclusion to be drawn. The fact that we were unable to study physiology in severe global ischaemia is testament to the fact that in clinical practice and using a cerebral perfusion pressure orientated approach, ischaemia is easier to control than hyperaemia. This suggests that we may be targeting perfusion pressures too high in some patients, and underscores the need to develop methods that allow optimisation of perfusion pressure in individual patients.

Our cerebral blood flow results disagree with work from Overgaard and Tweed. ${ }^{9}$ It is likely that the relatively small number of patients with predominantly normal cerebral blood flow has prevented us from establishing a correlation, and more data from patients with disturbed autoregulation are needed to characterise the relation between PRx and cerebral blood flow. In view of the large range of ages in this patient group, the analysis was repeated after correcting cerebral blood flow for age, using data from healthy volunteers and the same PET methods described above. However, this procedure did not improve the correlation. Another possible reason for our disagreement with Overgaard et al is that they used ${ }^{133} \mathrm{Xe}$ clearance, which predominantly measures blood flow in the 
cerebral cortex, whereas our method weights all parts of the brain equally. For technical reasons we could not measure PRx during the PET scan. While cerebral perfusion pressure, sedation, patient temperature, and arterial blood gases were maintained relatively constant, we cannot exclude changes in less obvious confounding variables. While these potential sources of error may have prevented us from observing a relation that was actually present, an alternative explanation is that we were studying a different, more specified population than Overgaard and Tweed. ${ }^{9}$ While their data spanned patients between two hours and 225 days after head injury, our patients were all studied within the first 11 days (mean 3.5 (2.3) days). It may well be that the relation between cerebral blood flow and dysautoregulation that they observed does not hold true in this acute phase. Furthermore, in our patients cerebral perfusion pressure was rigorously controlled, which was not the case in theirs. Alternatively, the absence of a correlation between cerebral blood flow and PRx could also suggest that pressure reactivity relates not to absolute cerebral blood flow levels but to their adequacy in the context of a given level of $\mathrm{CMRO}_{2}$.

The values for cerebral blood flow and $\mathrm{CMRo}_{2}$ given in table I may seem low, especially when considering that pressure reactivity was intact in the majority of patients. There are several reasons for these low values. First, the injury by itself will reduce $\mathrm{CMRO}_{2}$ and-where flow-metabolism coupling is intact-cerebral blood flow. ${ }^{22}$ All patients were sedated with propofol, which will lead to a further reduction in $\mathrm{CMRO}_{2}$ and blood flow ${ }^{25}$ without any untoward effects on autoregulation. ${ }^{24}$ However, it is also important to consider the accuracy of PET derived measures of cerebral blood flow and $\mathrm{CMRO}_{2}$. The steady state model we have used has been validated in healthy subjects ${ }^{26}$ and employed by PET centres in a variety of disease states, including acute stroke. ${ }^{27}$ The steady state model tends to give lower values for cerebral blood flow and $\mathrm{CMRO}_{2}$ than other techniques. ${ }^{28}{ }^{29}$ In addition, we report global values and do not segment into grey and white matter. It is well recognised that application of steady state PET models to such heterogeneous regions of interest can result in a significant underestimation of cerebral blood flow and $\mathrm{CMRO}_{2}$ of up to $20 \%{ }^{17}$ In these head injured patients, the PET and CT images did not provide adequate grey/white contrast, and segmentation of structural and functional images was substantially confounded by pathology. Finally, our values may have been affected by the choice of the blood-brain partition coefficient $(\rho)$. The lower the chosen value for $\rho$ the higher the cerebral blood flow and $\mathrm{CMRo}_{2}$ will be. Despite recent reports that have suggested a value of $0.86,{ }^{30}$ the true value is unknown, ${ }^{18}{ }^{31}$ particularly within diseased brain. We chose to use a $\rho$ of 0.95 based on the previous in vitro data. ${ }^{18}$ Despite PET being considered the gold standard for imaging cerebral blood flow and cerebral metabolism, the current models do not take into account the changes produced by pathophysiology in head injury and it is not possible to speculate on the error introduced by this limitation. However, none of these factors would result in changes that preclude comparisons within the context of our technique, and do not detract from the validity of our conclusions.

\section{Methodology for measurement of autoregulation}

Several techniques have been used to assess autoregulation in head injury, including the measurement of static $^{9}$ and dynamic $^{32}$ autoregulation in response to step changes in blood pressure. While these methods are well established, they may provide limited information on the response of the cerebral vasculature to smaller changes in cerebral perfusion pressure, which are more common and may be more clinically relevant. Further, they assess the cerebrovascular response at a single time point. We wished to relate PET derived variables to an index of autoregulatory capacity that addressed these issues, and chose a measure of cerebrovascular pressure reactivity for this purpose. The method we used for determination of pressure reactivity calculates PRx based on the continuous analysis of spontaneous slow waves of arterial blood pressure and intracranial pressure. Vascular pressure reactivity is central to concepts of autoregulation: changes in transmural pressure lead to adjustments in vessel diameter in order to keep cerebral blood flow constant. Pressure reactivity not only represents an important facet of autoregulation, but also has the advantage of being easily measured on-line. There is a strong association between pressure reactivity and autoregulation assessed using transcranial Doppler ultrasonography following head injury. ${ }^{10}$

\section{Outcome and clinical implications}

Our data confirm earlier work describing a correlation between PRx and outcome. ${ }^{14}$ The significant relation between high cerebral perfusion pressure and unfavourable outcome is in agreement with earlier retrospective work showing that managing patients at high perfusion pressures can lead to an unfavourable outcome..$^{23}$ We therefore need to address the question of whether overly aggressive augmentation of perfusion pressure may cause a decrease in the oxygen extraction fraction and therefore result in a less favourable outcome. We did not find a significant correlation between oxygen extraction fraction and cerebral perfusion pressure, nor was there an association between low oxygen extraction fraction and an unfavourable outcome. However, our study was neither designed nor powered for that purpose, and larger studies are necessary to address this important clinical question.

It is well recognised that autoregulatory efficiency depends on the level of cerebral perfusion pressure at which it is measured, and conventional intensive care management in head injury is based on increasing the perfusion pressure to a point where restoration of autoregulatory efficiency restores the balance between cerebral blood flow and cerebral metabolism. ${ }^{33}$ However, our data raise the intriguing possibility that dysautoregulation may be a consequence as well as a cause of cerebral metabolic failure. If these inferences were confirmed, the critical issue would be to identify patients in whom raising the cerebral perfusion pressure would restore cerebral metabolism to normal, and to differentiate them from those in whom metabolic failure is the primary process. This distinction will depend on the underlying mechanism of the relation. Three scenarios are possible.

The "traditional" model assumes that trauma leads to a disturbance in (microvascular) blood flow and endothelial function, which in turn cause pressure autoregulation to fail. The resultant instability of cerebral blood flow may lead to hyperaemia or ischaemia.

Alternatively, trauma may lead primarily to metabolic dysfunction, possibly reflecting mitochondrial impairment. ${ }^{34}$ In this instance the decrease in $\mathrm{CMRO}_{2}$ subsequently leads to dysautoregulation followed by unstable cerebral blood flow and further tissue injury.

Finally, it is possible that pressure reactivity and oxygen metabolism are not functionally related but simply reflect the trauma, with more severe trauma leading to more severe impairment of both variables. Our study was not designed to answer this question, as our data do not permit us to delineate clearly the temporal sequence of events in the pathophysiology of head injury.

Interventional studies that measure autoregulation, cerebral blood flow, and oxygen metabolism before and after step changes in cerebral perfusion pressure will be needed to address these issues. For clinical purposes, determining whether metabolic dysfunction is a cause or a result of disturbed pressure reactivity is important, as manipulating cerebral blood flow or shifting the patient to a more favourable part of the autoregulatory curve can only improve metabolism if the "traditional" model is predominant. Clear evidence that this was the case would strongly support development of an 
autoregulation oriented treatment strategy-that is, a strategy based on optimising cerebral perfusion pressure according to an index of autoregulation or pressure reactivity. On the other hand, if metabolic failure were the initial event we might need to focus on pharmacological interventions that improve metabolic (possibly mitochondrial) function. Experimental data provide potential candidates for such interventions. ${ }^{35-37}$

\section{Conclusions}

We have shown that dysautoregulation in head injury is associated with reductions in cerebral oxygen metabolism. In some patients, these associations occur in the context of reduced cerebral blood flow, a finding in keeping with previous investigations in this area. However, we have also demonstrated that in a substantial proportion of patients dysautoregulation is associated with primary metabolic impairment, characterised by $\mathrm{CMRO}_{2}$ reductions that cannot be attributed to ischaemia. This heterogeneity has implications for our understanding of pathophysiology and our choice of treatment in head injury. Monitoring pressure reactivity in conjunction with metabolic imaging may allow stratification of patients in whom individual disease mechanisms are dominant, allow patient selection for interventional trials using treatments aimed at autoregulation or metabolic dysfunction, and provide information on the response to such treatments. Further studies are needed to investigate the relation between outcome and cerebral oxygen metabolism after head injury.

\section{ACKNOWLEDGEMENTS}

We thank Professor Jean-Claude Baron for his advice on PET analysis. LAS is supported by a grant from the Margarete und Walter Lichtenstein-Stiftung (Basel, Switzerland), a Myron B Laver Grant (Department of Anaesthesia, University of Basel, Switzerland), by the Swiss National Science Foundation, and is recipient of an overseas research student award (Committee of Vice-Chancellors and Principals of the Universities of the United Kingdom). JPC is funded by a Wellcome research training fellowship and by a Beverley and Raymond Sackler studentship award. MC is on leave from the Warsaw University of Technology, Poland. PSM was supported by a clinical training fellowship from the Medical Research Council. This project was supported by the UK Government Technology Foresight Initiative, the Medical Research Council, and an equipment grant from the Royal College of Anaesthetists.

\section{Authors' affiliations}

L A Steiner, J P Coles, T D Fryer, F I Aigbirhio, J C Clark,

P Smielewski, T Donovan, Wolfson Brain Imaging Centre, University of Cambridge, Addenbrooke's Hospital, Cambridge, UK

M Czosnyka, P S Minhas, J D Pickard, Academic Neurosurgery, Addenbrooke's Hospital

D A Chatfield, D K Menon, University Department of Anaesthesia, Addenbrooke's Hospital

Competing interests: none declared

\section{REFERENCES}

1 Harris RJ, Symon L. Extracellular $\mathrm{pH}$, potassium, and calcium activities in progressive ischaemia of rat cortex. J Cereb Blood Flow Metab 1984;4:178-86

2 Bullock R, Sakas D, Patterson J, et al. Early post-traumatic cerebral blood flow mapping: correlation with structural damage after focal injury. Acta Neurochir Suppl (Wien) 1992;55:14-17

3 Compton JS, Teddy PJ. Cerebral arterial vasospasm following severe head injury: a transcranial Doppler study. Br J Neurosurg 1987;1:435-9.

4 Schröder ML, Muizelaar JP, Bullock MR, et al. Focal ischemia due to traumatic contusions documented by stable xenon-CT and ultrastructural studies. J Neurosurg 1995;82:966-71.

5 Kontos HA, Wei EP. Superoxide production in experimental brain injury. J Neurosurg 1986;64:803-7.

6 Armstead WM. Role of impaired cAMP and calcium-sensitive $\mathrm{K}+$ channel function in altered cerebral hemodynamics following brain injury. Brain Res 1997;768:177-84

7 Armstead WM. Brain injury impairs ATP-sensitive K+ channel function in piglet cerebral arteries. Stroke 1997;28:2273-9.

8 Petrov T, Rafols JA. Acute alterations of endothelin-1 and iNOS expression and control of the brain microcirculation after head trauma. Neurol Res 2001;23:139-43.
9 Overgaard J, Tweed WA. Cerebral circulation after head injury. 1 Cerebral blood flow and its regulation after closed head injury with emphasis on clinical correlations. J Neurosurg 1974;41:531-41.

10 Czosnyka M, Smielewski P, Kirkpatrick P, et al. Monitoring of cerebral autoregulation in head-injured patients. Stroke 1996:27:1829-34.

11 Lam JM, Hsiang JN, Poon WS. Monitoring of autoregulation using laser Doppler flowmetry in patients with head injury. J Neurosurg 1997;86:438-45

12 Czosnyka M, Smielewski P, Piechnik S, et al. Cerebral autoregulation following head injury. J Neurosurg 2001;95:756-63.

13 Bergsneider M, Hovda DA, Lee SM, et al. Dissociation of cerebral glucose metabolism and level of consciousness during the period of metabolic depression following human traumatic brain injury. $J$ Neurotrauma 2000;17:389-401.

14 Czosnyka M, Smielewski P, Kirkpatrick P, et al. Continuous assessment of the cerebral vasomotor reactivity in head injury. Neurosurgery 1997;41:11-17.

15 Jennett B, Bond $M$. Assessment of outcome after severe brain damage. Lancet 1975;i:480-4

16 Kinahan PE, Rogers JG. Analytic 3D image reconstruction using all detected events. Institute of Electrical and Electronic Engineers. Trans Nucl Sci 1989:36:964-8.

17 Baron JC, Frackowiak RS, Herholz K, et al. Use of PET methods for measurement of cerebral energy metabolism and hemodynamics in cerebrovascular disease. J Cereb Blood Flow Metab 1989;9:723-42.

18 Herscovitch $\mathbf{P}$, Raichle ME. What is the correct value for the brain-blood partition coefficient for water? J Cereb Blood Flow Metab 1985;5:65-9.

19 Studholme C, Hill DL, Hawkes DJ. Automated 3-D registration of MR and CT images of the head. Med Image Anal 1996;1:163-75.

20 Studholme C, Hill DL, Hawkes DJ. Automated three-dimensional registration of magnetic resonance and positron emission tomography brain images by multiresolution optimization of voxel similarity measures. Med Phys 1997;24:25-35.

21 Marshall LF, Marshall SB, Klauber MR, et al. The diagnosis of head injury requires a classification based on computed axial tomography. $J$ Neurotrauma 1992:9:S287-92.

22 Obrist WD, Langfitt TW, Jaggi JL, et al. Cerebral blood flow and metabolism in comatose patients with acute head injury. Relationship to intracranial hypertension. J Neurosurg 1984;61:241-53.

23 Steiner LA, Czosnyka M. Piechnik SK, et al. Continuous monitoring of cerebrovascular pressure reactivity allows determination of optimal cerebral perfusion pressure in patients with traumatic brain injury. Crit Care Med 2002;30:733-8.

24 Strebel S, Lam AM, Matta B, et al. Dynamic and static cerebral autoregulation during isoflurane, desflurane, and propofol anesthesia. Anesthesiology 1995;83:66-76.

25 Oshima T, Karasawa F, Satoh T. Effects of propofol on cerebral blood flow and the metabolic rate of oxygen in humans. Acta Anaesthesiol Scand 2002;46:831-5

26 Frackowiak RS, Lenzi GL, Jones T, et al. Quantitative measurement of regional cerebral blood flow and oxygen metabolism in man using ${ }^{15} \mathrm{O}$ and positron emission tomography: theory, procedure, and normal values. J Comput Assist Tomogr 1980;4:727-36.

27 Marchal G, Benali K, Iglesias S, et al. Voxel-based mapping of rreversible ischaemic damage with PET in acute stroke. Brain 1999:122:2387-400.

28 Kanno I, Lammertsma AA, Heather JD, et al. Measurement of cerebral blood flow using bolus inhalation of $\mathrm{C}^{15} \mathrm{O}_{2}$ and positron emission tomography: description of the method and its comparison with the $\mathrm{C}^{15} \mathrm{O}_{2}$ continuous inhalation method. J Cereb Blood Flow Metab 1984;4:224-34.

29 Kanno I, lida H, Miura S, et al. A system for cerebral blood flow measurement using an $\mathrm{H} 2150$ autoradiographic method and positron emission tomography. J Cereb Blood Flow Metab 1987;7: 143-53.

30 Lammertsma AA, Martin AJ, Friston KJ, et al. In vivo measurement of the volume of distribution of water in cerebral grey matter: effects on the calculation of regional cerebral blood flow. J Cereb Blood Flow Metab 1992;12:291-5.

31 Lammertsma AA, Jones T, Frackowiak RS, et al. A theoretical study of the steady-state model for measuring regional cerebral blood flow and oxygen utilisation using oxygen-15. J Comput Assist Tomogr $1981 ; 5: 544-50$

32 Newell DW, Aaslid R, Stooss R, et al. Evaluation of hemodynamic responses in head injury patients with transcranial Doppler monitoring. Acta Neurochir 1997;139:804-17.

33 Rosner MJ, Rosner SD, Johnson AH. Cerebral perfusion pressure: management protocol and clinical results. J Neurosurg 1995;83:949-62

34 Verweij BH, Muizelaar JP, Vinas FC, et al. Impaired cerebral mitochondrial function after traumatic brain injury in humans. J Neurosurg 2000:93:815-20.

35 Xiong Y, Peterson PL, Muizelaar JP, et al. Amelioration of mitochondrial function by a novel antioxidant U-101033E following traumatic brain injury in rats. J Neurotrauma 1997; 14:907-17.

36 Xiong $\mathrm{Y}$, Peterson PL, Verweii $\mathrm{BH}$, et al. Mitochondrial dysfunction after experimental traumatic brain iniury: combined efficacy of SNX-1 11 and U-101033E. J Neurotrauma 1998;15:531-44.

37 Verweij BH, Muizelaar JP, Vinas FC, et al. Improvement in mitochondrial dysfunction as a new surrogate efficiency measure for preclinical trials: dose-response and time-window profiles for administration of the calcium channel blocker Ziconotide in experimental brain injury. $J$ Neurosurg 2000;93:829-34. 